\title{
Wavefront calibration testing of the James Webb Space Telescope primary mirror center of curvature optical assembly
}

\author{
Gene Olczak $^{\mathrm{a}^{*}}$, Conrad Wells ${ }^{\mathrm{a}}$, David J. Fischer ${ }^{\mathrm{b}}$, Mark T. Connolly ${ }^{\mathrm{a}}$, \\ aTT Exelis Geospatial Systems, 400 Initiative Drive, P.O. Box 60488, Rochester, NY, USA 14606 \\ 'TASC, 4801 Stonecroft Blvd, Chantilly VA 20151-3822
}

\begin{abstract}
The James Webb Space Telescope (JWST) Optical Telescope Element (OTE) consists of a 6.6 meter clear aperture, allreflective, three-mirror anastigmat ${ }^{1}$. The 18 -segment primary mirror (PM) presents unique and challenging assembly, integration, alignment and testing requirements. A full aperture center of curvature optical test is performed in cryogenic vacuum conditions at the integrated observatory level to verify PM performance requirements. Two wavefront calibration tests are utilized to verify the low and Mid/High spatial frequency performance of the test system. In this paper the methods and results of the wavefront calibration tests are presented.
\end{abstract}

\section{BACKGROUND}

The JWST primary mirror will be tested at Johnson Space Center in a cryogenic vacuum chamber using the vertical test configuration illustrated in Figure 1.

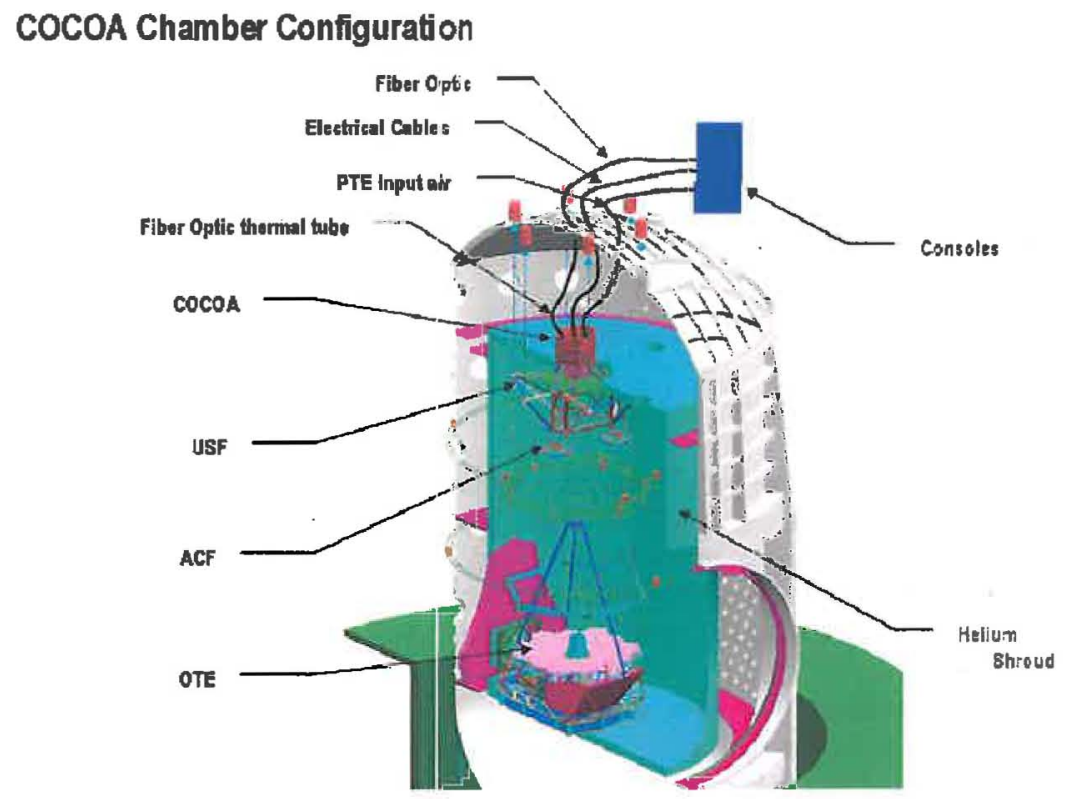

Figure 1 The cryogenic test configuration.

The optical layout of the Center of Curvature $(\mathrm{CoC})$ test set configuration for the JWST primary mirror test is shown in Figure 2. In Figure 2 a) the PM is shown as a simple monolith at the bottom of the figure and the null assembly is at the top. The spot and field definitions are such that the PM is treated as the object and the image is formed at the interferometer sensor. The aperture of the internal relay is the spot of the system (This aperture is conjugate to the front focus of the objective lens where its image has a diameter of $1.5 \mathrm{~mm}$ ). Figure $2 \mathrm{~b}$ ) is the view zoomed to show the null in more detail (with the PM paraxial focus (center of curvature) at bottom). Figure $2 \mathrm{c}$ ) shows the system from the 
objective lens front focus up. The internal relay depicted here is an approximation developed prior to receiving the vendor design information.

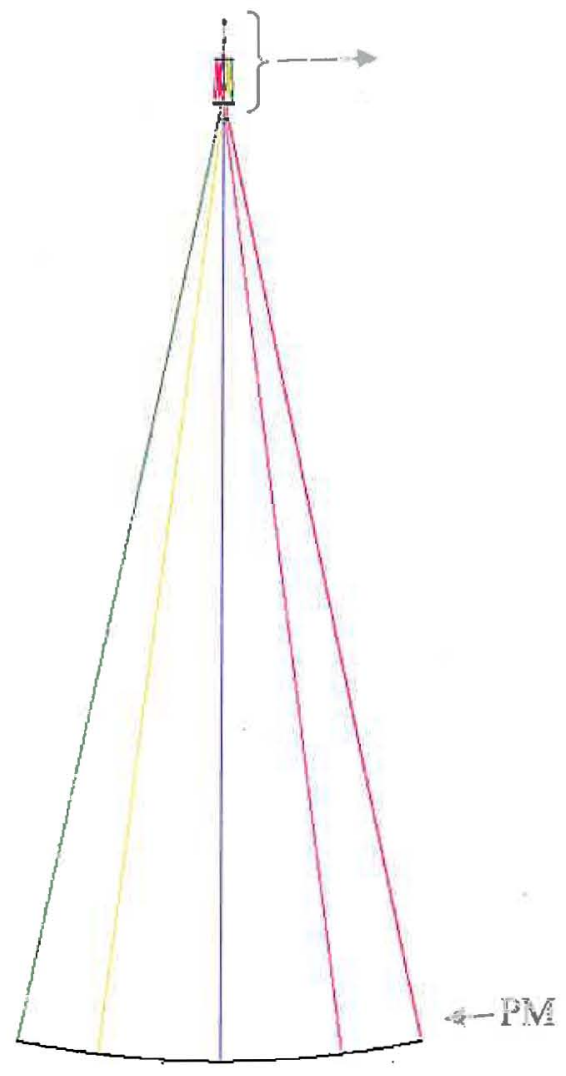

a) PM to Sensor
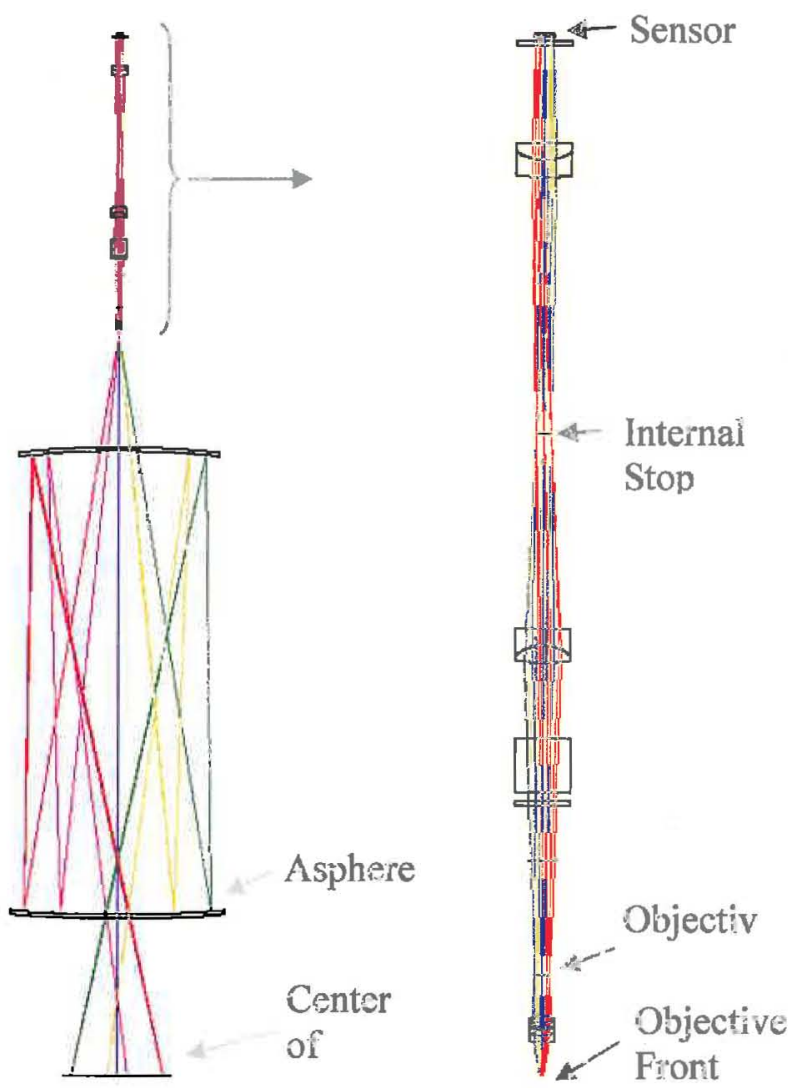

c) Front Focus to Sensor

Figure 2 PM imaging in the test configuration.

The imaging properties of the novel optical null provide the low distortion, low alignment sensitivity and achromatic properties make it the best choice for this test application compared to other optical null choices ${ }^{2}$. Because the optical null includes an aspheric mirror the calibration of middle and high spatial frequency errors is of particular concern. Such parts have the potential for manufacturing and test artifacts that are not usually present in conventionally fabricated optics. The rest of the optical null is conventionally manufactured and therefore lower risk.

For the purposes of describing PM imaging, the configuration shown in Figure 2 is single pass. In other words, each surface in the ray trace is impinged only once. The single pass optical imaging performance for the system of Figure 2 is reported in Figure 3. Here one can see that the PM is diffraction limited as has less that $1 \%$ distortion. 

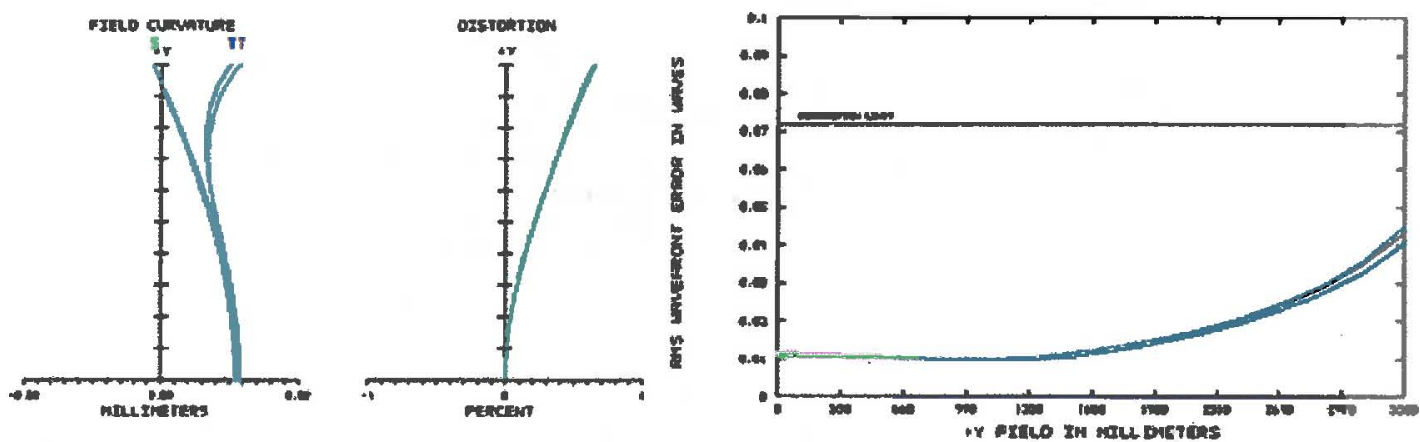

Figure 3 Single pass imaging performance to interferometer sensor.

When performing a PM test all surfaces except the PM are impinged twice i.e. double pass. For simplicity only the image back to the interferometer entrance port will be considered for the double pass analysis. Here the object field is defined as points on the outbound entrance port plane (from the interferometer) and the image surface is defined on the inbound entrance port plane (to the interferometer). The rays passes through or off of every surface in the COCOA optical path then off of the PM and then back though the COCOA (this is a sequential model so there are first and second instances of each COCOA surface). The imaging performance is shown in Figure 4. Here there is some degradation but again one can see a well behaved system. The extremely low distortion is evidence that the system can image the pupil back on itself.
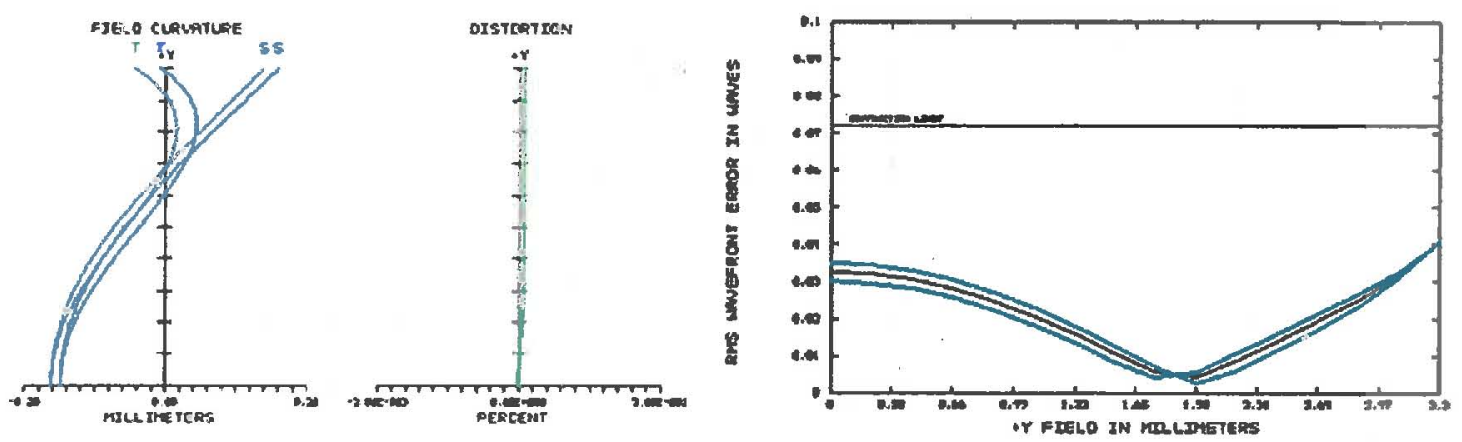

Figure 4 Double pass imaging performance to the interferometer entrance port.

\section{THE SWFT CALIBRATION CONFIGURATION}

\subsection{CGH theory of operation}

A computer generated hologram (CGH) is placed at or near the PM CoC for the System Wavefront Test (SWFT) as shown in Figure 5. The CGH acts as an inverse null lens: it returns light to the objective lens focus with no wavefront variance if everything is perfect (at the $687 \mathrm{~nm}$ wavelength only). The CGH design is determined by the surface projection from the PM surface toward the CGH diffractive surface. Other than choice of operating wavelength the CGH design is independent of the the null and interferometer design. The CGH wavefront return is encoded in a second order power carrier. Due to the CGH location at the edge of the PM caustic, the wavefront returned is very aspheric (Greater than $2 \mathrm{~mm}$ aspheric departure from the best fit sphere). 


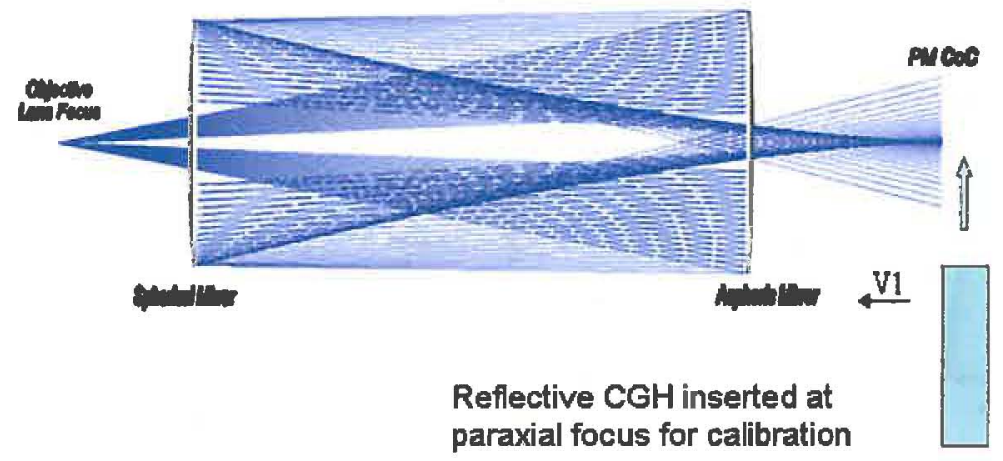

Figure 5 COCOA Null and SWFT CGH.

\subsection{SWFT configuration double pass imaging performance}

The analysis in this section is similar to that is Section 1 except here the PM is replaced with the CGH. The CGH is located at the $\mathrm{CoC}$. The $\mathrm{CGH}$ is not pupil conjugate and while the chief rays for the finite conjugate analysis have little retrace error, the marginal rays are significantly different than those returned by the PM in the test configuration. As a result, the interferometer pupil is not well imaged back on itself as shown in Figure 6.
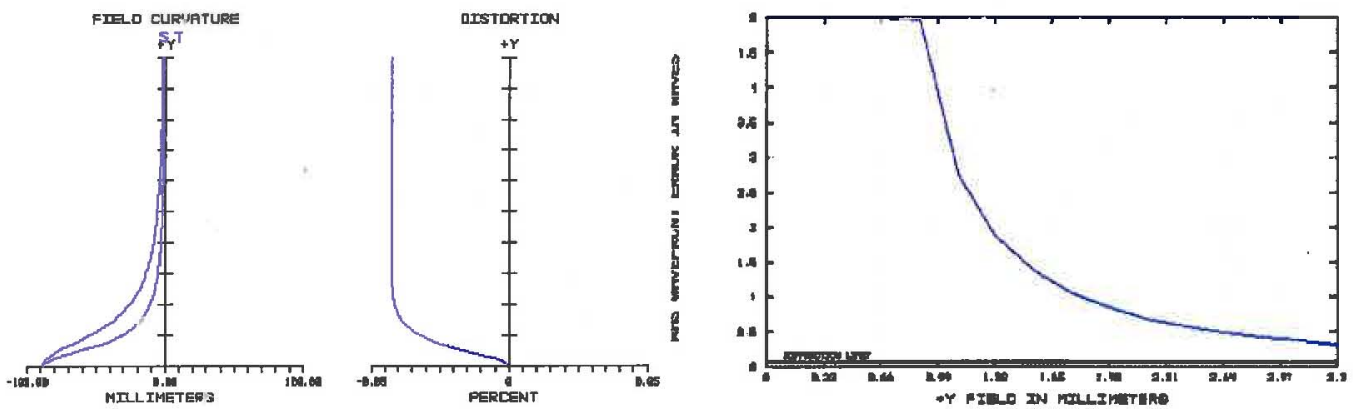

Figure 6 Zemax analysis of pupil imaging performance with a SWFT calibration CGH.

Here the field curvature is very large on axis (This effect increases as the CGH design location is made closer to the CoC.). This is not too much of a concern since the system is obscured on axis. However focus is shifted significantly everywhere. It is lowest at the edge of the pupil image field: -0.5 millimeter for the tangential plane and -1.4 millimeter for the sagittal plane. This is large compared to the depth of focus ( 0.6 millimeters).

\subsection{SWFT configuration double pass phase imaging analysis}

The SWFT is analyzed using a method for predicting the degradation in the visibility (measured wavefront amplitude) of period sinusoidal phase patterns ${ }^{3}$. The method is based on Talbot imaging and (for small wavefront amplitudes) a transfer function $(T F)$ is derived. For a phase variation of period $d, T F$ is the observed amplitude $W^{\prime}$ divided by the actual amplitude $W$ as shown in Equation 1 . Here $\mathbf{z}$ is the distance from best focus and $\lambda$ is the wavelength (all length units must be common).

$$
T F=\frac{W}{W}=\cos \left(\frac{\pi \lambda z}{d^{2}}\right)
$$

(From Reference 3) (1)

Figure 7 shows the $T F$ effects predicted for the first pass asphere using defocus due to field curvature as shown in Figure 6 (where focus is always maintained on the second pass) in radial pupil coordinates. The wavelength is $0.687 \mathrm{um}$ and 
the PM image (aperture image) has a 6600 um diameter. Three periods are plotted but annotated in terms of cycles over the PM image aperture (c/a): $5 \mathrm{c} / \mathrm{a}$ (the transition between low and mid spatial frequencies), $30 \mathrm{c} / \mathrm{a}$ (the transition between mid and high spatial frequencies) and $80 \mathrm{c} / \mathrm{a}$ (the highest spatial frequency in the test requirements. The values of $T F$ to the left of the vertical dashed line (Obsc Thresh) are of less interest since this is inside the missing center PM segment.

The results show good performance ( $T F$ close to 1 ) at low spatial frequencies but very poor results for the mid and high spatial frequencies. This is especially true the closer the field is to on-axis. The second pass $T F$ is essentially unity at all frequencies and fields. For double pass calibration the total measured phase error due to the asphere will be determined by the sum of the first pass and second pass transfer functions for all fields and frequencies.

Sagittal Image Phase Transfer Function versus Pupil Field

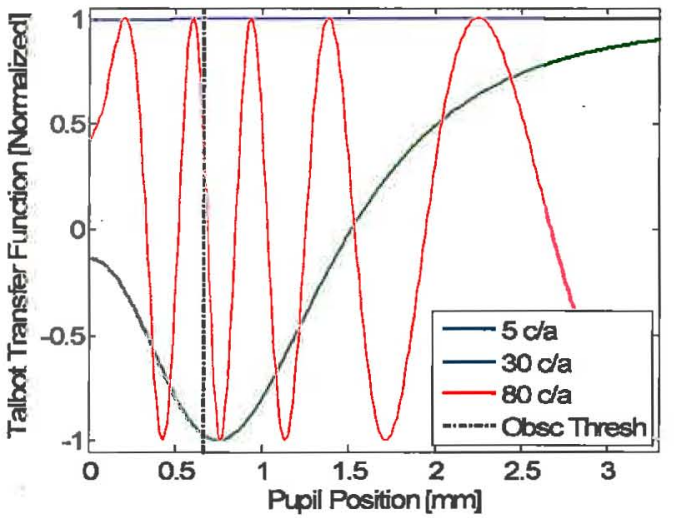

Tangential Image Phase Transfer Function versus Pupil Field

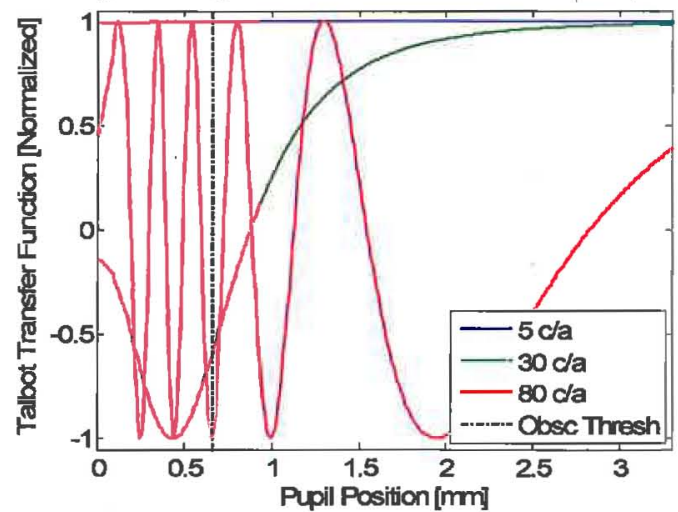

Figure 7 SWFT pupil imaging phase transfer function with a CGH: object surface at the first pass of the asphere.

\section{THE I-SWFT CALIBRATION CONFIGURATION}

Due to the limitations of the CGH depicted above, a second auxiliary calibration optical system was developed ${ }^{4}$. This system uses a plano convex imaging lens between the optical null and CGH such that a pupil image is formed on the CGH and pupil imaging performance is equivalent to the test configuration. This imaged CGH (I-SWFT) configuration is depicted in Figure 8. In order to keep cost low the I-SWFT includes an off-the-shelf lens. The CGH for the I-SWFT is smaller and less asperic than the SWFT CGH and utilizes a first order power carrier.

The I-SWFT is not considered for use as the primary calibration tool and due to its larger footprint (compared to the SWFT) would be impractical to implement for in situ calibration in the Johnson test. Instead, the planned approach is to use the I-SWFT to capture middle to high spatial frequency features of the null optics. These errors are dominated by the asphere surface that is not expected to vary over time. The low order time varying errors are captured as needed by the SWFT.

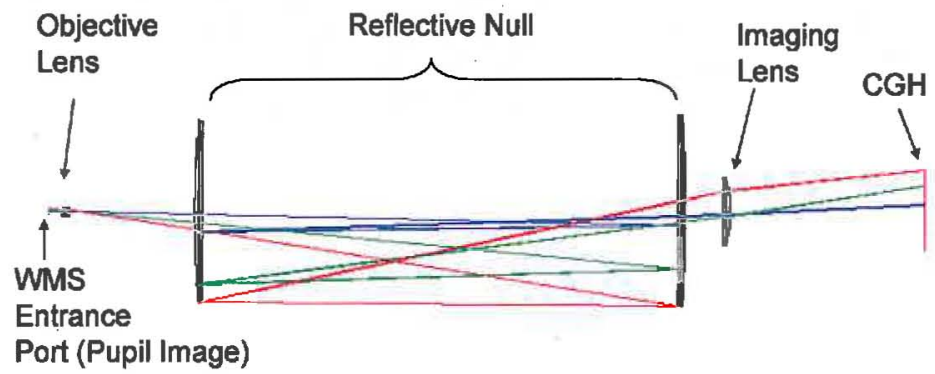

Figure 8 COCOA optical null and I-SWFT 


\section{CALIBRATION TEST RESULTS}

The JWST COCOA was integrated at a Micro Automated System Group in Rochester NY. ITT Exelis personnel performed optical testing during the integration process (though the facility was not designed for this purpose). The wavefront recording instrument for the optical null is a 4D Technologies, Inc. multi-wavelength interferometer ${ }^{5}$. This interferometer has near-simultaneous dual wavelength capture capability. This capability allows for the dis-ambiguous determination of piston between primary mirror segments even in the.presence of vibration. The two wavelength data set is analyzed as a synthetic wavelength that is determined by the difference between the two wavelengths of the two lasers yielding discrete values between 16.8 microns and 15 millimeters. Data processing of fringe images for mirror-segmentfigure and pose estimation is performed using custom software written in the MATLAB software application ${ }^{6}$.

The objective lens used for the test is designed to have a minimal wavefront error (as built single pass wavefront is less than $7 \mathrm{~nm}$ RMS) over an extended wavelength range $(660-687 \mathrm{~nm})$ and also provide a high resolution image of the primary mirror to the interferometer?

The testing completed to date provides a baseline for the performance of the null in ambient loosely controlled environments (as opposed to the tightly controlled Johnson test). As such these tests represent a first look at the calibration tests while final performance tests are pending.

\subsection{SWFT results}

In this test, the SWFT calibration hologram is precisely aligned to the COCOA optical null and utilized to measure the COCOA wavefront. The CGH is rotated about the optical axis at 8 orientations to average out asymmetric error in the CGH. At each orientation a 100 frame average is acquired.

The Zernike aberration predictions are sensitive to the normalizing radius used for the fit. The SWFT CGH is designed so that there is a defined end to the diffraction pattern at the outer CGH edge that is visible in the interferometer and is mapped to the PM. In practice, the fringe modulation image was found to provide the best detail of this feature when compared to a fringe or intensity (sum of fringes) image. The three image types are depicted in Figure 9.

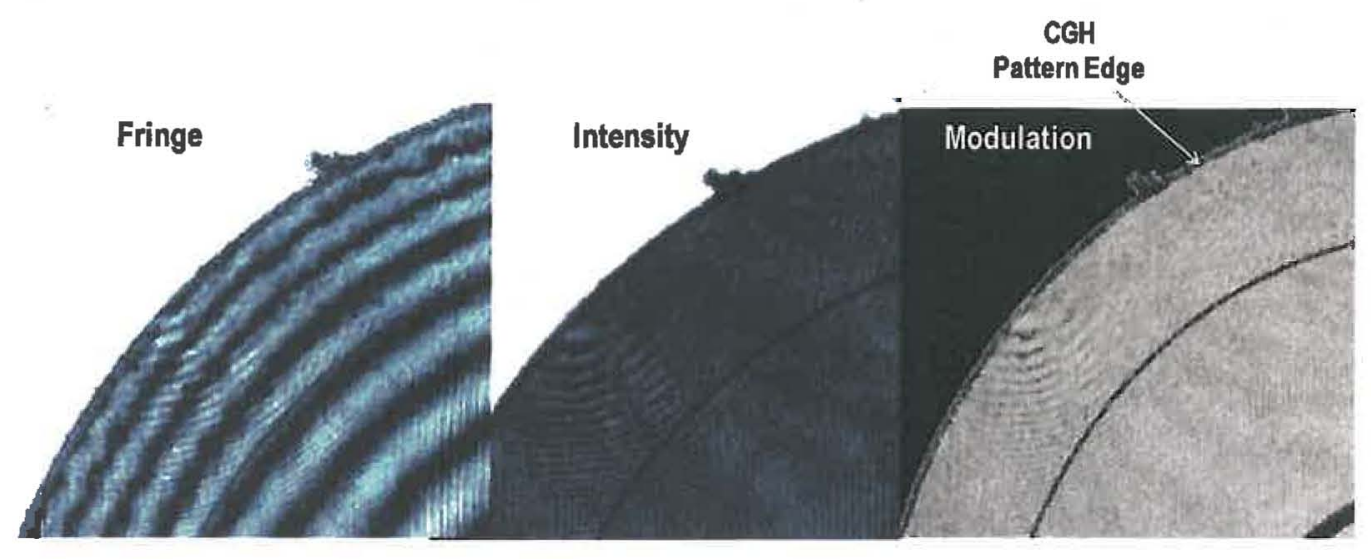

Figure 9 Images of a portion of the $\mathrm{CGH}$ in test (upper left quadrant).

The SWFT CGH is very sensitive to wavelength and has a linear dependence between wavelength and spherical aberration. Spherical aberration, power and vertex distance are the parameters that determine the deviation of the as-built best fit conic PM from the nominal design. Two SWFT CGH configurations were planned for the JWST COCOA: The first for ambient air (standard pressure and temperature) and the second for vacuum. Figure 10 shows the relationship between wavelength in nanometers (nm) and the Zernike spherical aberration for the as-built null with an ambient CGH. The results are plotted for the tunable laser, a fixed offset laser (with repetitions) and the optical model prediction. 


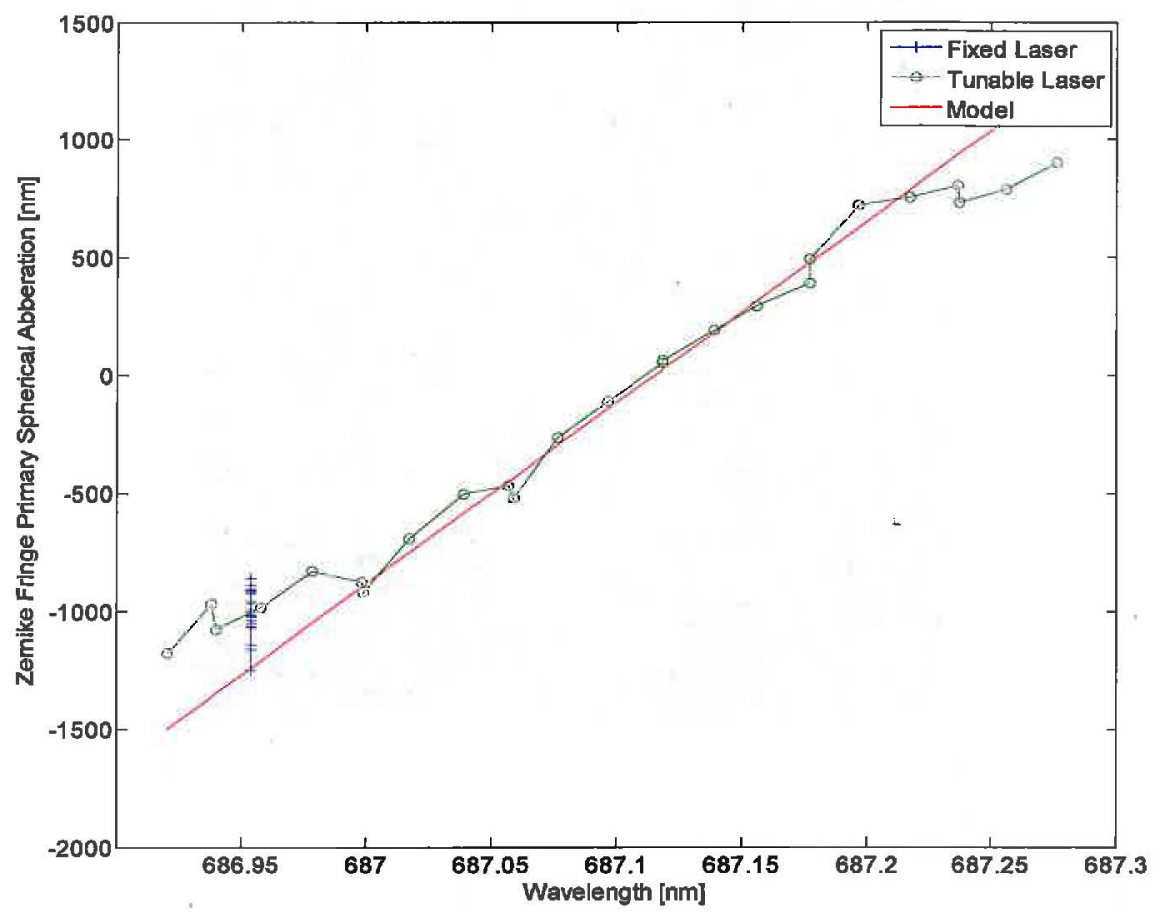

Figure 10 Spherical aberration versus vacuum wavelength at a fixed $\mathrm{CGH}$ position.

The results in the previous figure are of particular interest since, although the calibration CGHs are designed to operate at the fixed wavelength (the primary laser in the interferometer being non-tunable), it has subsequently been discovered that the primary laser wavelength varies considerably more than expected. Thus a choice is to be made between operating with a considerable wavelength bias or using the secondary tunable laser for calibration. In the latter case there is less bias (fewer fringes) but the $4 \mathrm{D}$ acquisition software as not designed to work in the mode so data taken is more cumbersome and time consuming.

The following wavefront analysis is considered with coma, tilt, power and spherical aberrations removed. Coma and tilt are dependent on the alignment of the CGH to the optical null (and controlled to limit these errors to tens of nanometers). Power and spherical aberration are booked in the the radius and conic error budget and considered separately from PM figure error. A wavefront map of the 100 frame average eight orientation test is shown in Figure 11. The total wavefront error in the as-built null optics is $83 \mathrm{~nm}$ RMS and is dominated by mirror mount trefoil, asphere test artifacts and marks from small tool manufacturing. The masked annulus in the map is due to a thin fiducial line on the CGH surface. Distortion mapping at the C.GH significantly broadens this feature in the interferometer.

Note that there is little sign of assembly contributors to the as-built wavefront error of the optical null. As noted earlier, the optical null design is very insensitive to alignment. Further, due to the stringent stability requirements of some of the JWST tests, the entire optical null structure is design for minimal thermal expansion. There is some temperature sensitivity in the interferometer with respect to astigmatism. In general, the interferometer drives the temperature operating range requirements. 


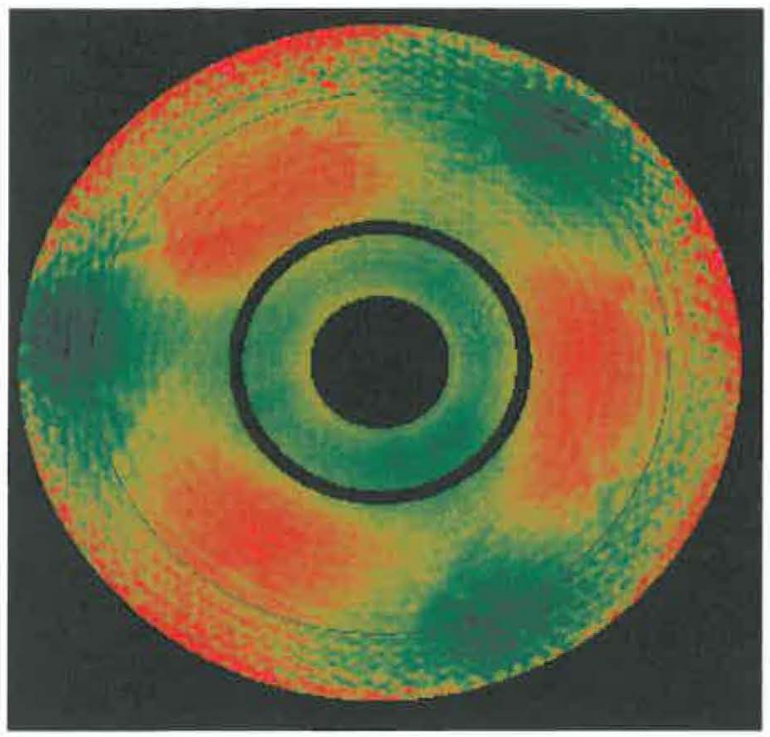

Figure 11 Wavefront error map of the null optics at a $687.15 \mathrm{~nm}$ test wavelength.

The wavefront is spatially filtered into low spatial frequencies (LF), mid spatial frequencies (MF) and high spatial frequencies (HF) as depicted in figure 12. Here one can see mirror mount distortion dominating the low spatial frequency map and component test artifacts dominating the mid spatial frequency map. The component test for the asphere was performed with a partial aperture CGH at multiple orientations. Stitching artifacts from this process are now permanent features of the asphere that will not vary during the test. Despite the limitations of the imaging properties of this test, the asphere tooling artifacts are easily visible in the high spatial frequency map (these same features are visible in the part print provided by the asphere manufacturer, as are the stitching artifacts).

There is good agreement between the tunable laser measured wavefront and fixed laser measured wavefront. This is demonstrated by analysis of eight orientation data sets where the tunable laser data (tuned to the CGH design wavelength, see figure 10) and fixed laser data were captured simultaneously. The CGH was positioned so that power and spherical aberration were minimized for the tunable laser. In this case there is a $1420 \mathrm{~nm}$ RMS power and spherical bias on the fixed laser data. In this condition the difference between the two measurements for all other wavefront error contributors is $7 \mathrm{~nm}$ RMS (including coma).

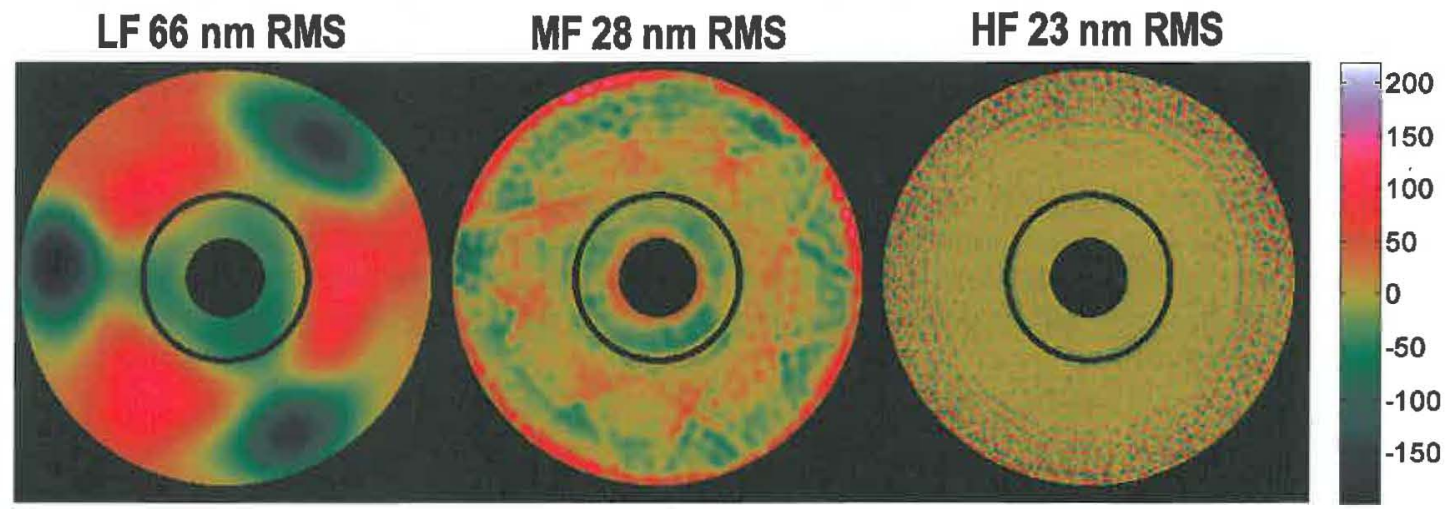

Figure 12 Spatially filtered components of the wavefront of the as-built null optics from the SWFT. 


\subsection{I-SWFT Results}

The I-SWFT was conducted after the initial SWFT was completed. The I-SWFT procedures were more difficult to perform than those for the SWFT. The primary difficulty was that the refractive index melt data for the off-the-shelf lens had a significant error. This resulted in an improper mapping of the null optic wavefront onto the CGH and a corresponding increase in high order spherical aberration. To counter this, the test wavelength and lens to CGH spacing were adjusted to reduce the as-tested wavefront. The mount design (intended primary for the SWFT) made alignment difficult and large temperature variations in the test environment (sometimes greater that 10 degrees Celsius per day) resulted in drift in the the measurements (the I-SWFT uses a low cost aluminum structure to space the lens to the CGH). The results for an eight orientation 100 frame average test are depicted in Figure 13. Here the results are filtered by spatial frequency as in Figure 12.

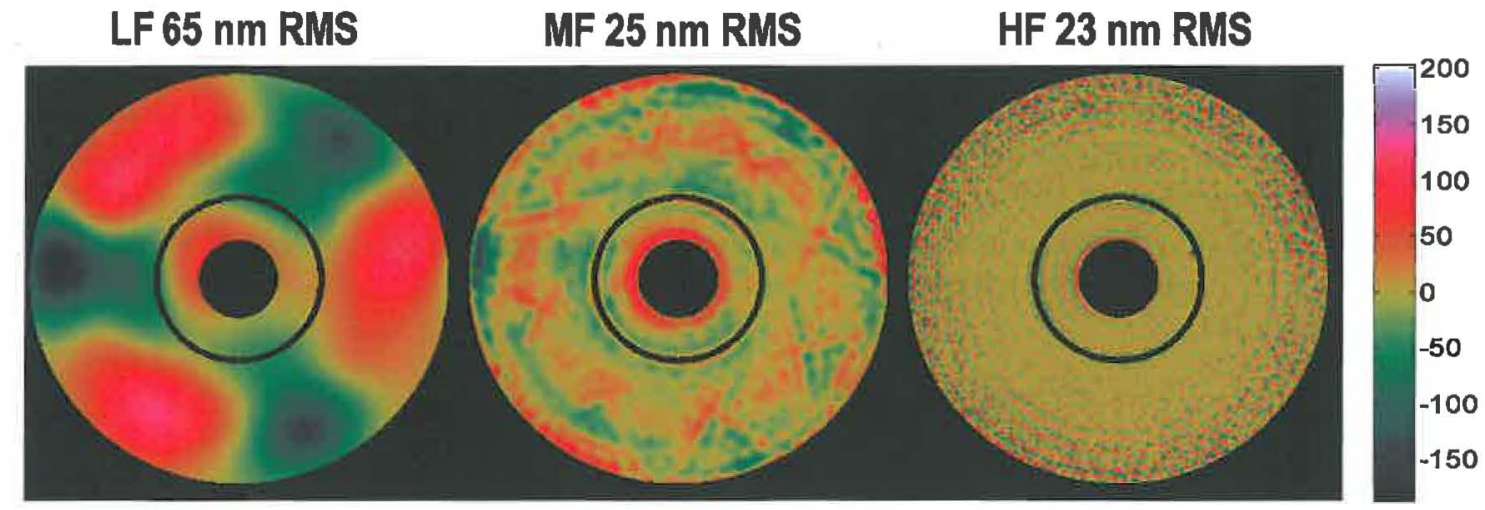

Figure 13 Spatially filtered components of the as-built null optics from the I-SWFT.

At a glance the CGH and I-SWFT data is in good agreement. As shown in figure 14, the difference map of the CGH minus I-SWFT is dominated by spherical aberration in the mid and low spatial frequencies. There are small differences in the high spatial frequency map. The form of these differences is such that the features grow more distinct toward the optical axis, as might be expected given the Talbot imaging analysis. Analysis of the difference in the high spatial frequency content of the two tests is on-going.

LF 36 nm RMS

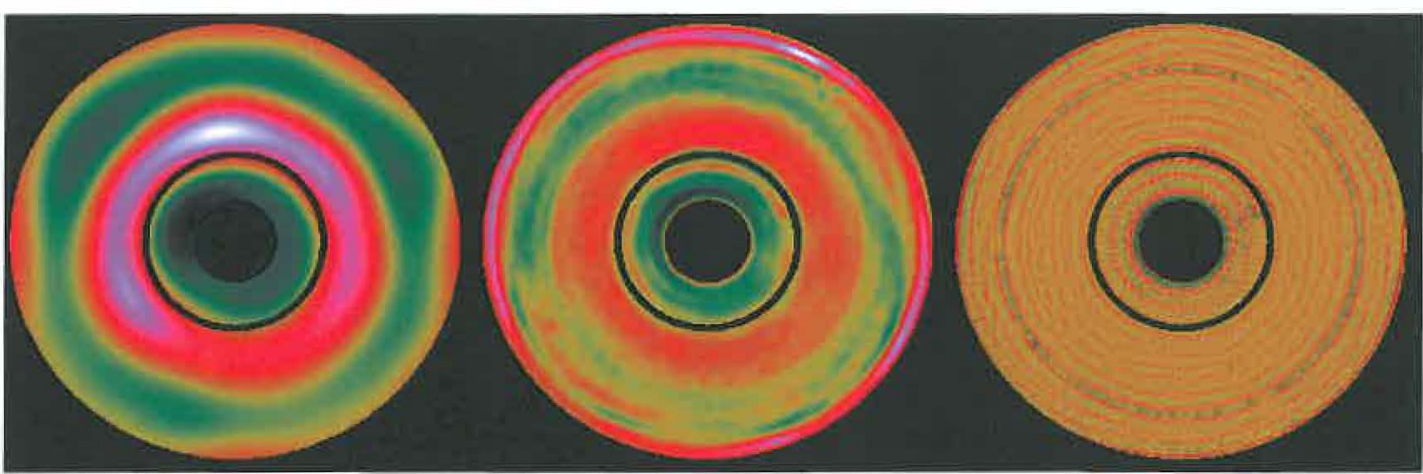

Figure 14 Spatially filtered difference map components of the as-built null optics tests (SWFT minus I-SWFT).

HF 8 nm RMS

\section{CONCLUSION}

In this paper the two wavefront calibration tests are utilized to verify the low and $\mathrm{mid} / \mathrm{high}$ spatial frequency performance of the JWST PM optical null test system is described. The test results to date are presented. Initial results are promising but limited by temperature variations that substantially exceeded the operating requirements of the interferometer. Further tests in air and vacuum are on-going at Marshall Space Flight Center. Further analysis against test uncertainty requirements will be deferred until these tests are complete. 


\section{REFERENCES}

[1] Sabelhaus, Phillip A., Campbell, Doug, Clampin, Mark, Decker, John, Greenhouse, Matt, Johns, Alan, Menzel, Mike, Smith, Robert, Sullivan, Pam, "An overview of the James Webb Space Telescope (JWST) project, . of SPIE Vol. 5899, pp. 241-254 (2005).

[2] Olczak, Eugene G. , Hannon, John J., Dey, Thomas W., Jensen, Arthur E., "Optical nulling apparatus and method for testing an optical surface", US Patent 7336370 (2008).

[3] Zhao, Chunyu and Burge, James H., "Imaging aberrations from null correctors", Proc. of SPIE Vol. 6723, 67230L, (2007).

[4] Olczak, Eugene G., Pending US Patent (Allowed by the USPTO, May 2012).

[5] North-Morris, Michael B., Millerd, James E., Brock, Neal J. and Hayes, John B., "Phase-shifting multiwavelength dynamic interferometer", Proc. SPIE 5531, 64 (2004).

[6] Olczak, Gene, Fischer, David J., Connolly, Mark, Wells, Conrad, “James Webb Space Telescope primary mirror integration: testing the multiwavelength interferometer on the test bed telescope", Proc. of SPIE Vol. 8146, pp. 814608-814608, (2011).

[7] Olczak, Eugene G., "Objective lens simultaneously optimized for pupil ghosting, wavefront delivery and pupil imaging", US Patent 7876505 (2008). 